\title{
Los contactos del español con lenguas amerindias y europeas de migración en la clase de ELE
}

\section{Mário Domingues Cruz}

\section{(2) OpenEdition}

1 Journals

Edición electrónica

URL: https://journals.openedition.org/cher/849

DOI: $10.4000 /$ cher.849

ISSN: 2803-5992

\section{Editor}

Presses universitaires de Strasbourg

\section{Edición impresa}

Fecha de publicación: 12 diciembre 2019

Paginación: 63-81

ISBN: 979-10-344-0057-7

ISSN: 1968-035X

\section{Referencia electrónica}

Mário Domingues Cruz, «Los contactos del español con lenguas amerindias y europeas de migración en la clase de ELE», reCHERches [En línea], 23 | 2019, Publicado el 22 septiembre 2021, consultado el 17 noviembre 2021. URL: http://journals.openedition.org/cher/849 ; DOI: https://doi.org/10.4000/cher. 849 


\title{
Los contactos del español con lenguas amerindias y europeas de migración en la clase de ELE
}

\author{
MÁrIo Domingues CRUZ1
}

\begin{abstract}
T a lengua es un rasgo distintivo de pertenencia social e incluso (inter)cultural. Como sabemos, el contacto entre lenguas a lo largo del tiempo ha generado diferentes fenómenos de intercambio e influencia (Moreno Fernández 2010).

Con relación a la variación lingüística, hay que tener en cuenta tanto los factores sociolingüísticos, como la trayectoria histórica de un idioma, ya que el estudio de la variación diacrónica de la lengua nos permite entender mejor los mecanismos y procesos lingüísticos inherentes al cambio sincrónico (Klee y Lynch 2009). Estas variedades se deben, en parte, a las diferencias lingüísticas que los colonos llevaron desde la Península, al mestizaje y, más tarde, a la influencia del sustrato y, posteriormente, del superestrato (Moreno Fernández 2010).

Ante las demandas de la educación del siglo xxi en nuestra sociedad globalizada (P21 2015), es indispensable no solo integrar la diversidad lingüística en nuestros discursos y prácticas en la clase de Español como Lengua Extranjera (ELE), sino también reflexionar en el aula sobre los cambios que han ocurrido en el español debido a los contactos que han existido con otras lenguas.

En la primera parte de este artículo vamos a analizar el fenómeno de contacto de lenguas en el continente americano entre el español (lengua de conquista), otras de origen indígena (quechua, guaraní, maya, etc.) y otras lenguas europeas (como el italiano), y cómo esto se puede trasladar a la clase de ELE. En la segunda parte se presentará un estudio de caso relacionado con las variedades lingüísticas y culturales de Hispanoamérica; en concreto, se dará cuenta de propuestas didácticas que plasman un trabajo relacionado con las influencias de las lenguas de contacto.
\end{abstract}

1 Mário Domingues Cruz, Politécnico do Porto - Escola Superior de Educação, Universidad de Vigo, inED, CIDTFF ORCID: 0000-0001-8894-8821 


\section{El primer contacto con lenguas amerindias}

Como sabemos, el español es la lengua materna de gran parte de los ciudadanos de países latinoamericanos. Según Company Company (2016), el español de esta región se caracteriza por una gran variación lingüística diacrónica y sincrónica, y por una diferenciación dialectal. Sin embargo, estos dialectos presentan rasgos comunes que permiten a los hablantes de esta región comunicarse sin grandes dificultades mediante el español, lengua que es producto de la influencia de las lenguas amerindias ya presentes en el territorio en la época precolombina y de otras lenguas que llegaron al Nuevo Mundo a través de diversos flujos migratorios.

De acuerdo con Sánchez-Albornoz (2006), la primera presencia del español en el continente americano está relacionada con movimientos migratorios interregionales (heteroglósicos primero; homoglósicos después), debidos a las políticas comerciales y coloniales de España, así como a razones socioeconómicas de los propios emigrantes. En este momento cruzaron el Atlántico 200000 españoles. Entre 1830 y 1935, 350000 españoles se establecieron en tierras americanas. En el último tercio del siglo xIX y el primero del siglo xx fueron más de 3 millones los emigrantes de nacionalidad española que viajaron hacia América (véase la Figura 1).

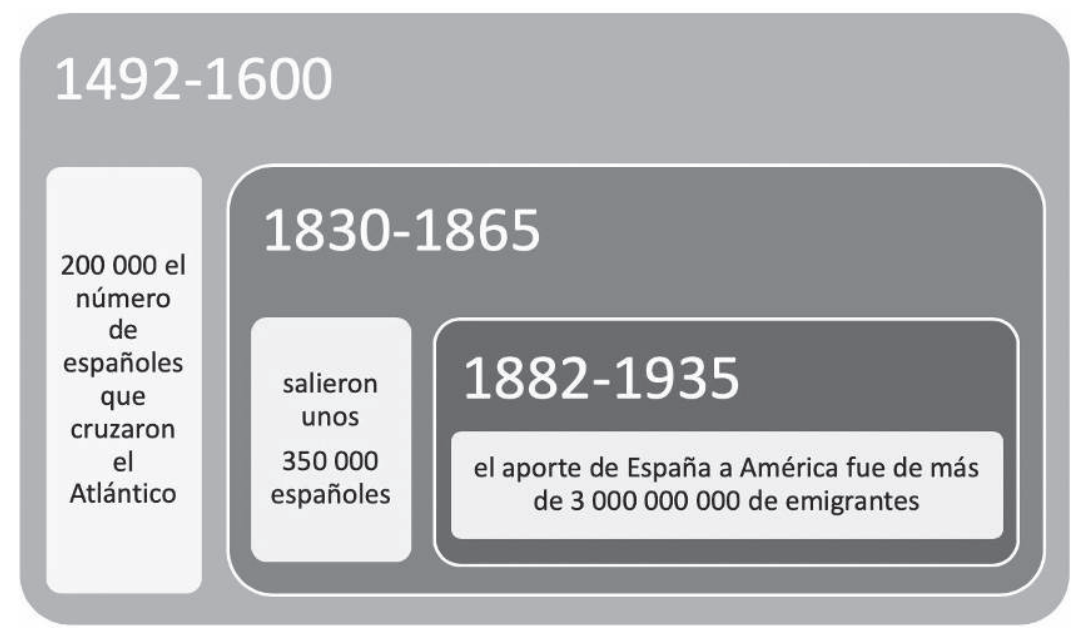

Figura 1: Movimientos migratorios de España hacia América a lo largo de los siglos (cf. Boyd-Bowman 1976, Sánchez-Albornoz 2006).

De hecho, el primer contacto lingüístico en América entre los pueblos indígenas de la región y los europeos surge en 1492, durante los viajes de Cristóbal Colón. Durante la etapa inicial de expansión y colonización, los Austrias determinaron que los indios frecuentaran las escuelas y que muchos de sus misioneros y súbditos aprendieran las lenguas amerindias. En aras de llevar a cabo esta labor, se crearon gramáticas y diccionarios de los diferentes 
idiomas indígenas. De hecho, este primer contacto lingüístico promovió la incorporación del léxico indígena al español, ante la necesidad de nombrar cosas $\mathrm{u}$ objetos desconocidos por los europeos. Algunas de estas palabras aparecen documentadas en el Diario de Colón. De hecho, en su primer viaje, Cristobal Colón llegó a las islas de Cuba y La Española, donde tuvo contacto con el taíno, un idioma caribeño. En este diario encontramos palabras como canoa, hamaca, cacique, batata, bahía, yuca, maíz, iguana y huracán. También se hizo más tarde la traducción de obras literarias prehispánicas como Popol Vuh, como resalta León-Portilla (2002).

Víctimas de enfermedades y masacres, resultado de los contactos con los conquistadores, se redujo el número de taínohablantes y de hablantes de otros dialectos, llegando incluso a desaparecer, dejándonos, sin embargo, algunas palabras de sus idiomas en el español. Durante la conquista, se perdieron unas 1750 lenguas en Latinoamérica. Además, se debe señalar que hubo una fuerte presión social y cultural para usar el español como lengua franca.

Tabla 1: Las lenguas indígenas en Latinoamérica (adaptado de Klee y Lynch 2009).

\begin{tabular}{|l|l|l|c|}
\hline \multicolumn{1}{|c|}{ Lengua } & \multicolumn{1}{c|}{ Familia } & \multicolumn{1}{c|}{ Países } & N de hablantes \\
\hline quechua, quichua & quechua & $\begin{array}{l}\text { Perú, Bolivia, Ecuador, Colombia, } \\
\text { Argentina }\end{array}$ & 8500000 \\
\hline guaraní & tupi-guaraní & Paraguay, Bolivia, Argentina, Brasil & 4000000 \\
\hline aimara & jaki & Perú, Bolivia & 2200000 \\
\hline náhuatl & uto-azteca & México & 1200000 \\
\hline zapoteco & sapoteka & México & 387000 \\
\hline mixteco & misteka & México & 387000 \\
\hline mapuche & araucania & Chile, Argentina & 300000 \\
\hline
\end{tabular}

La diversidad de idiomas y dialectos amerindios, el bajo número de colonos y la ausencia de contactos directos entre los distintos grupos en contextos urbanos (los contactos en ámbitos rurales eran más frecuentes) son las razones que justifican la dificuldad que encontraron los españoles para castellanizar a los nativos del nuevo continente.

En ese momento, como podemos ver en la tabla 1, las lenguas indígenas más importantes en Latinoamérica eran el quechua, el guaraní, el aimara y el náhuatl.

Teniendo en cuenta nuestro corpus, que recoge la influencia de las lenguas náhuatl, quechua, guaraní e italiano, en el próximo apartado vamos a prestar atención a los contactos que se llevaron a cabo entre el español y cada uno de estos idiomas, analizando sus rasgos principales (léxicos, fonéticos y morfosintácticos), así como las influencias que tuvieron sobre el castellano. 


\section{El contacto de lenguas en la Nueva España: el caso del náhuatl}

En 1519, Cortés llegó a México y se enteró de que había un gran imperio en el centro del territorio gracias a los contactos iniciales que mantuvo con los grupos mayas. Enseguida, Cortés llevó a cabo una campaña militar y logró conquistar Tenochitlán y los territorios que esta ciudad dominaba, subyugando a los aztecas.

Cuando los españoles llegaron a la región de México, se hablaban más de ochenta lenguas y dialectos diferentes dentro del imperio azteca, pero el idioma del comercio y del gobierno era el náhuatl. Como vimos en el apartado anterior, los colonos no mantuvieron el náhuatl como lengua oficial, pero tampoco lograron que el castellano lo desplazara. Sin embargo, algunos investigadores, como Parodi (2006), consideran que los mestizos, criollos y algunos colonos sí que hablaban el náhuatl, pero con diferentes matices.

En la actualidad, el español es el idioma de unidad en México. Con la ley de instrucción obligatoria de 1875, el español alcanzó un estatus más importante al ser reconocido como:

la lengua nacional, no la española pura, sino la española modelada por nuestro medio físico y social, por los restos de las civilizaciones a medias desaparecidas y por las creaciones que en México ha hecho surgir la mutua compenetración de las razas (Castillo 1965: 109).

De esta manera, se dio cuenta de la herencia amerindia y de la labor conjunta entre las distintas razas.

Vamos ahora a prestar atención a los cambios que ocurrieron como resultado de los contactos del español con el náhuatl. No olvidemos que, en la actualidad, este último lo hablan unos dos millones de habitantes en México, Guatemala y El Salvador.

Según Heath (1972), muchos préstamos léxicos del náhuatl que designaban plantas, animales, comida y prendas de vestir eran desconocidos para los colonos $y$, por lo tanto, ingresaron en el español general. Entre los más importantes hay que señalar chocolate, peyote, tomate, guacamole, coyote, mole, guajalote, quetzal, petaca, cuate, guarache, papalote (cf. Klee y Lynch 2009).

Respecto a los rasgos fonéticos, Lopes Blanch (1967) indica que hay cinco características fonéticas principales que se han atribuido a la influencia del náhuatl, que resumimos en la Figura 2. Con respecto a los tres primeros rasgos, solo los encontramos en voces de origen indoamericano, como en los ejemplos: mixiote, Quetzalcóatl y nantli. Con relación a la pérdida de vocales átonas, este fenómeno se observa en palabras como p'scar (pescar), croksi (creo que sí), trescient's pes's (trescientos pesos) (cf. Lope Blanch 1967). En lo que se refiere al último rasgo, al aprender el español, los náhuatlhablantes lo reemplazaban por el fonema /ts/, lo cual le daba más tensión (cf. Klee y Lynch 2009), aunque la tesis del sustrato con relación a este fenómeno no está totalmente comprobada (cf. Canfield 1934). 


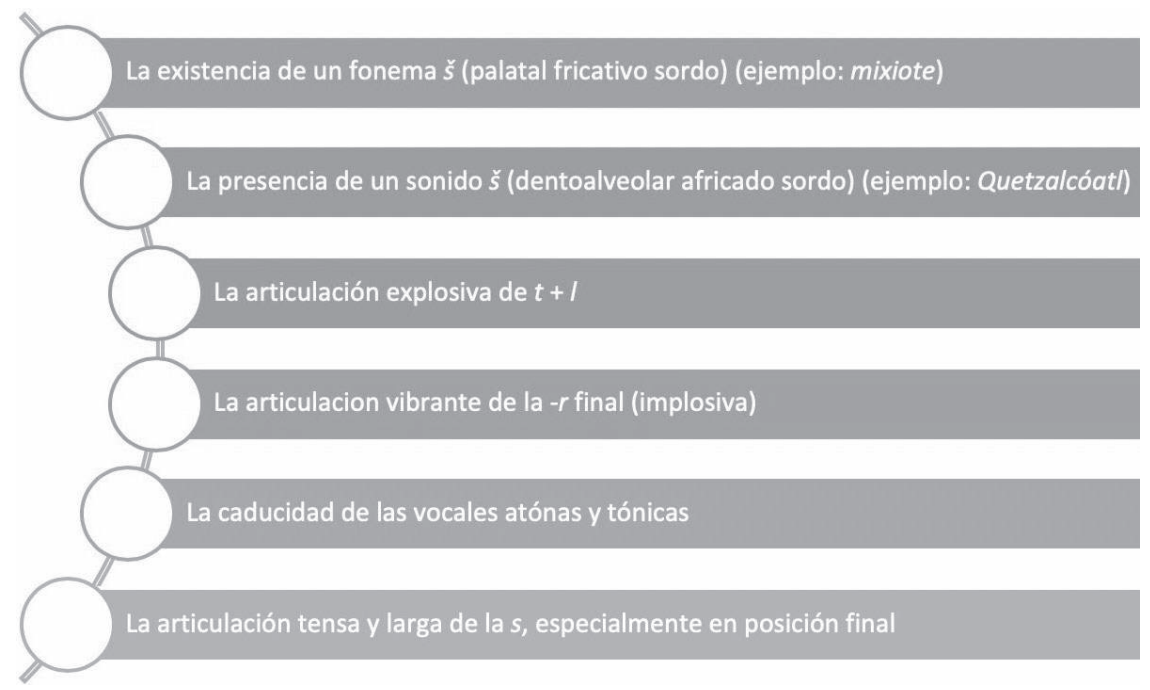

Figura 2: Principales características fonéticas atribuidas a la influencia del náhuatl (adaptado de Lope Blanch 1967).

A nivel morfosintáctico, Flores Farfán (1998) nota varias características que se pueden atribuir a la transferencia del náhuatl, pero aquí resaltamos solo tres de ellas. La primera es la transferencia de la pluralización posesiva del náhuatl, por ejemplo, para decir la casa de ellos, estos hablantes siguen el modelo nahua y dicen sus casa. La segunda es el uso redundante de lo, debido a una transferencia del náhuatl, en el que hay que marcar obligatoriamente la transitividad: ¿No lo vieron mi llave? (Klee y Lynch 2009: 121). La tercera tiene que ver con la preferencia por los verbos modales y del progresivo en lugar del presente simple, ya que el náhuatl es un idioma más rico en estos (cf. Lockart 1991). Por último, debemos señalar el uso de aunque, con el significado de pase lo que pase, propio del náhuatl.

\section{El contacto de lenguas en el Cono Sur (los casos del quechua, del guaraní y del italiano)}

A continuación, nos centraremos en el estudio de los contactos producidos entre el español, el quechua y el guaraní en el Cono Sur, al mismo tiempo que analizaremos la influencia del italiano en el español de esta región.

El quechua se habla, mayoritariamente, en Bolivia, Colombia, Ecuador y Perú, coincidiendo con las regiones del antiguo imperio inca. Actualmente, lo hablan aproximadamente siete millones de personas. Hay distintos dialectos del quechua en la zona andina, algunos de los cuales no son mutuamente inteligibles. De hecho, las variedades peruanas y bolivianas se denominan quechua, mientras la colombiana se llama inda y la ecuatoriana quichua. 
La influencia léxica del quechua se ha extendido a toda Latinoamérica, sobre todo en lo referido a palabras que designan grupos humanos, animales, plantas y la geografía de la zona andina (cf. Azorín Fernández 2008). A modo de ejemplo, cabe mencionar: cóndor, inca, llama, mate, pampa y papa; y otras palabras, cuyo uso se restringe al Cono Sur, como chacra (granja), choclo (mazorca), puna (tierra alta), guagua (bebé), poroto (judía), entre otras (cf. Klee y Lynch 2009).

En lo que se refiere a los rasgos fonéticos, la variación vocálica es característica entre los quechuahablantes que han adquirido el español como segunda lengua: a) confusión entre vocales medias y altas debido a transferencias del quechua: [lebro] por libro (Cerrón Palomino 1987; Klee y Lynch 2009); b) reducción de vocales átonas, sobre todo en contacto con /s/ como en oyent(e)s (Lipski 1994; Klee y Lynch 2009); c) yeísmo (Gómez 2003; Klee y Lynch 2009).

Si hablamos de aspectos morfosintácticos, el español de la región se caracteriza por los siguientes rasgos: a) uso del doble posesivo, como en su familia de usted o su libro de María; b) uso del gerundio como verbo principal (de ahí vuelta tranquilo manejando así) o como complemento de verbos específicos (entrando de la calle así ve va); c) uso no normativo del pretérito perfecto en el habla informal, en contextos en que se esperaría el uso del pretérito indefinido, como en el ejemplo El año 1956, creo, ha muerto; d) ausencia del pronombre clítico la en el discurso u omisión total de los pronombres clíticos, como en los ejemplos La escuela lo realicé en el Colegio Malinche y Aquí en Arequipa (-) aprendí yo solo (cf. Klee y Lynch 2009).

Con respecto al guaraní, hay que decir que es una lengua muy antigua, que, actualmente, es idioma oficial en Paraguay y se enseña en las escuelas. A lo largo de los siglos XVI y xVII, los jesuitas escribían en guaraní para evangelizar a la población amerindia durante sus misiones, por lo que acabó convirtiéndose en uno de los pocos idiomas amerindios con prestigio social.

Klee y Lynch (2009) dan cuenta de los siguientes aspectos con relación a los contactos entre el español y el guaraní: por un lado, se ha constatado un fuerte influjo léxico y una moderada influencia morfosintáctica del castellano en el guaraní; por otro, el guaraní ha aportado algunas palabras de la flora y la fauna locales al español. De acuerdo con Lipski (1994), el guaraní ha aportado algunos vocablos al español, que se conocen fuera de la región, tales como: ñandú, urubú (buitre), mita’i (niño), topará (parra), caigüe (perezoso), yagua (delator).

Si prestamos atención al componente fonético, hay varias particularidades del español de esta región que, en principio, se podrían atribuir a la influencia del guaraní, como por ejemplo: a) la nasalización del vocalismo; b) el uso de la sexta vocal guaraní (cerrada centro-posterior no labializada) en vocablos castellanos, como en el ejemplo puerta, que se pronuncia [pierta]; c) la tendencia a la inserción de la oclusión glotal entre vocales, como en alcohol [alko?ol]; d) la realización de $m$ en variación con $n$ alveolar como en melón [melóm] (Lipski, 1994). 
En lo referido a la influencia morfosintáctica del guaraní en el español, debemos citar el leísmo, que funciona en el español paraguayo como única forma de objeto directo para la totalidad de los casos posibles sintácticamente, independientemente del género, del número y de la caracterización. También es frecuente la omisión del pronombre clítico: La profesora de cuarto curso ya no da eso; no toca. También se produce la doble negación: Nada no dije. Además, se utiliza el artículo indefinido con posesivo: Ese mi amigo ya no vive aquí, y la preposición en con verbos de movimiento: Voy en Asunción (cf. Granda 1980).

Para terminar, identificaremos los contactos del español del Cono Sur con el italiano, en tanto que lengua europea de migración. Como vemos en la Tabla 2, entre 1861 y 1920 llegaron más de dos millones de italianos a Argentina. La mayoría de los italianos se estableció en ciudades argentinas (Buenos Aires, sobre todo).

La combinación entre dos factores, la proximidad entre el español y el italiano (inteligibilidad mutua entre los dos idiomas), por una parte, y el hecho de que los inmigrantes italianos hablaban variedades regionales del italiano y no se entendían perfectamente, favoreció la rápida adquisición del español y el rápido abandono del italiano.

Se crearon dos dialectos con la presencia de los italianos. El primero, el cocoliche, que se consideraba un español chapurreado, una jerga híbrida, con una interlengua muy variable, que hablaba el gaucho italiano. Teruggi (1978: 183) lo caracterizó de la siguiente manera: «Se trata de una cadencia especial, una música del idioma, un arrastre de los fonemas y un ritmo particular, que los oyentes del interior del país identifican como un canto o tonada».

Tabla 2: Inmigración italiana en Argentina (adaptado de Fontanella de Weinberg 1992).

\begin{tabular}{|c|c|c|c|}
\hline Período & $\begin{array}{c}\text { Inmigración total } \\
\text { en Argentina }\end{array}$ & $\begin{array}{c}\text { Inmigración italiana } \\
\text { en Argentina }\end{array}$ & $\%$ \\
\hline $1861-1870$ & 159570 & 113554 & 71 \\
\hline $1871-1880$ & 260885 & 152061 & 58 \\
\hline $1881-1890$ & 841122 & 493885 & 59 \\
\hline $1891-1900$ & 648326 & 425693 & 57 \\
\hline $1901-1910$ & 1764103 & 796190 & 45 \\
\hline $1911-1920$ & 1204919 & 2270525 & 29 \\
\hline
\end{tabular}

Luego, tenemos el lunfardo de la región porteña, en Buenos Aires, que se hablaba en los barrios del Río de la Plata y se consideraba el lenguaje de los ladrones, de los delincuentes. Algunas voces del lunfardo son bacán (persona rica), cana (policía), mina (mujer), morfar (comer) y pibe (chaval). Además, hay que resaltar que existen muchos préstamos del lunfardo en la variedad del español rioplatense. A nivel fonológico, «el español de Buenos Aires se caracteriza por un descenso entonativo al final de la frase que es mucho más pronunciado que en otras variedades del español» (Colantoni 2004). 


\section{El enfoque comunicativo experiencial y una (hiper)pedagogía de discursos}

Una vez terminado nuestro análisis de los influjos de los contactos entre el español y los idiomas del sustrato amerindio y del sustrato europeo de inmigración, vamos ahora a ver qué enfoques metodológicos y estrategias hemos movilizado en nuestro estudio práctico, prestando especial atención al enfoque comunicativo experiencial y a la (hiper)pedagogía de los discursos que hemos implementado en nuestras prácticas de enseñanza de ELE.

En nuestros días, la creación de conocimiento no se restringe al aula. También fuera de las cuatro paredes, con la relación que nuestros alumnos crean con el mundo global, marcadamente digital, se crea conocimiento. Así, se involucran en un proceso comunicativo con hispanohablantes procedentes de todas partes. Creemos que hay que repensar los procesos de enseñanza de ELE bajo una pedagogía de los discursos, a través de la cual los alumnos se enfrentan a fenómenos lingüísticos y culturales, que les activen su «encendido emocional» (Mora 2013). Hablamos aquí de enfoques y metodologías experienciales, es decir, que ponen a nuestro alumnado en contacto con tareas activas y enlazadas con sus necesidades y entornos presenciales y digitales, en las cuales se ponen en contacto con textos auténticos, muestras vivas de las variedades del español.

Por ello, esta pedagogía de los discursos, que se desarrolló a finales de los años setenta y a lo largo de los años 80 con Joaquim Fonseca y Fernanda Irene Fonseca $(1977,1986)$, tiene en cuenta la centralidad del texto o del discurso en el aula de lenguas, con el objetivo de promover en el alumno la conciencia de la existencia de recursos múltiples en el idioma y llevarlo a la exploración de los mismos (Fonseca 1986). Asimismo, la pedagogía de los discursos permite la articulación de dos componentes muy importantes en nuestro estudio: el estudio de la lengua y de textos literarios (Fonseca 2000) e hipermedia.

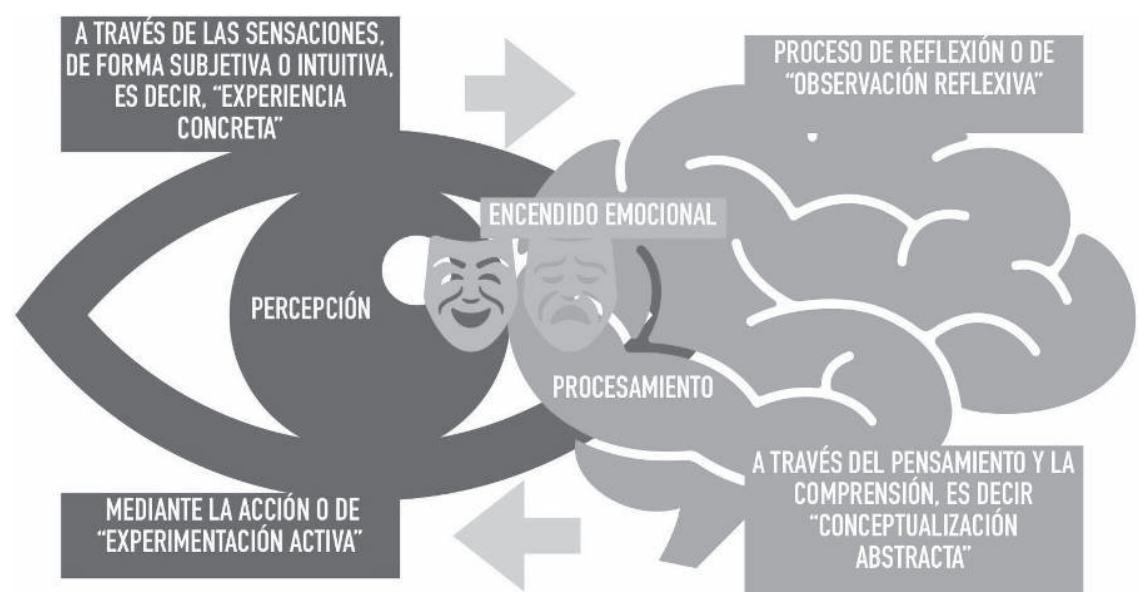

Figura 3: El encendido emocional, la percepción y el procesamiento de la experiencia (Shams y Seitz 2008; adaptado de Mora 2013). 
En este contexto, como resaltamos anteriormente, creemos que se deben abordar prácticas lingüísticas y comunicativas que despierten el «encendido emocional» (Mora 2013) del alumnado. Esta dimensión emocional promueve la conexión con su propia identidad y con la de los otros y, además, con sus experiencias previas y con el mundo afectivo de las sensaciones, lo que hace que se mantengan involucrados en una tarea en el aula. Como vemos en la Figura 3, el componente afectivo facilita el desarrollo del «personal input, initiative, and selfdirection in the learning process» (Knutson 2015: 54) [aporte personal, iniciativa y autodirección en el proceso de aprendizaje], por lo que podemos decir que las experiencias proporcionadas por el aprendizaje a través de recursos holísticos e hipermedios permiten actividades de reflexión y estimulación del análisis crítico, para que los alumnos puedan asimilar y crear conocimientos proactivos que van a ser movilizados en otros contextos (cf. Fernández-Corbacho 2014; Foncubierta y Rodríguez 2015).

De hecho, el enfoque comunicativo experiencial (Fernández-Corbacho 2014) busca que la enseñanza de las lenguas sea proactiva, eficaz, marcante y muy conectada a la vida real. Sus cuatro principios son la enseñanza activa, el uso auténtico de la lengua, la personalización y la afectividad, por lo que se deben rechazar estrategias algo repetitivas, mecánicas y aisladas de la vida de nuestros alumnos. Así pues, el alumnado debe tener la oportunidad de implicarse en tareas casi reales en entornos que les resulten familiares.

Veamos ahora nuestras propuestas didácticas, que tienen en cuenta estos enfoques y pedagogías, y que plasman el trabajo con el tema de las lenguas de contacto con el español en la clase de ELE.

\section{Propuestas didácticas que plasman un trabajo relacionado con las influencias de las lenguas de contacto en el español}

Con respecto al corpus principal de nuestro estudio, tuvimos en cuenta el análisis de discursos literarios e hipermedia, con relación a las variedades lingüísticas y culturales del español latinoamericano que ofrecen dichos recursos. Además, bajo una perspectiva metodológica etnográfica y cualitativa, analizamos cuestionarios ${ }^{2}$, fichas de trabajo creadas por nosotros y también los proyectos producidos por una muestra de 61 alumnos lusohablantes de enseñanza superior de E/LE de los niveles A2/B1 de la región de Oporto.

Las cuestiones de investigación de nuestro macro-estudio relacionado con el tema de las variedades del español son las siguientes: 1. ¿Qué representaciones

2 Los doscuestionarios fueron creados para el propósito de un macro-estudio, relacionado con las variedades lingüísticas y culturales del español americano, que incluye diferentes fenómenos lingüísticos y aspectos culturales de las distintas regiones. El cuestionario, que se centra en los aspectos sociobiográficos para inventariar las representaciones sociolingüísticas y socioculturales de los estudiantes con relación al mundo hispánico está disponible en línea: <https://goo.gl/forms/FIhtPtsGrCG2w5p22>. 
tienen los estudiantes lusohablantes de E/LE sobre el español en contacto con otras lenguas? y 2. ¿Qué discursos literarios e hipermedia y sus rasgos pueden facilitar el trabajo con este tema?

Con relación a nuestra primera cuestión de investigación, no vamos a analizar las respuestas de manera detallada. Elegimos algunas preguntas relacionadas con el tema de este artículo. La primera es «¿Cuántos idiomas/dialectos se hablaban antes de la llegada de los colonos españoles?». En el Gráfico 1, vemos que la mayoría de los alumnos contesta 20 , por lo que se revela un desconocimiento de los números reales de la diversidad de idiomas que se hablaban antes de la llegada de los españoles.

Respecto a la segunda pregunta formulada, «Elige las palabras que crees que son de origen hispanoamericano», al analizar el Gráfico 2, vemos que gran parte de los alumnos no saben cuáles son las palabras que tienen un origen hispanoamericano y que están en el español a causa de los contactos de lengua que ocurrieron. Hay palabras como tabaco, huracán, caimán, tequila y azúcar que casi la mitad de los estudiantes consigue identificar. No obstante, la mayoría de los estudiantes no parece tener conocimientos sobre el tema.

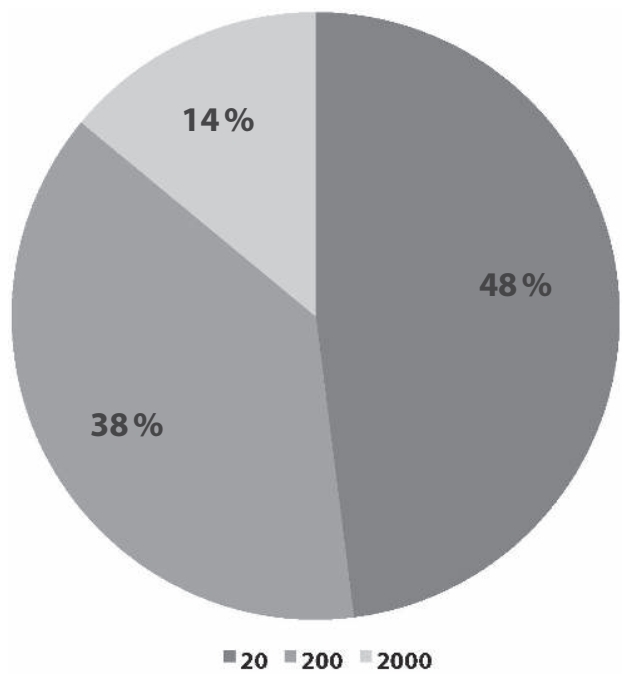

Gráfico 1: Respuestas a la pregunta ¿Cuántos idiomas/dialectos se hablaban antes de la llegada de los colonos españoles?

Poniendo nuestra atención en las necesidades reales del alumnado y profesorado, y teniendo en cuenta estudios previos, como por ejemplo los de Altmann y Vences (2004) y Anadón Pérez (2003), elegimos algunos recursos literarios e hipermedia que nos parecieron adecuados, creando propuestas didácticas con materiales relacionados con muestras vivas de las culturas de los países hispanoamericanos, a través de los cuales los alumnos se pudieron involucrar en el estudio de las variedades del español, y específicamente en el estudio de los contactos que el español tuvo con otros idiomas. 


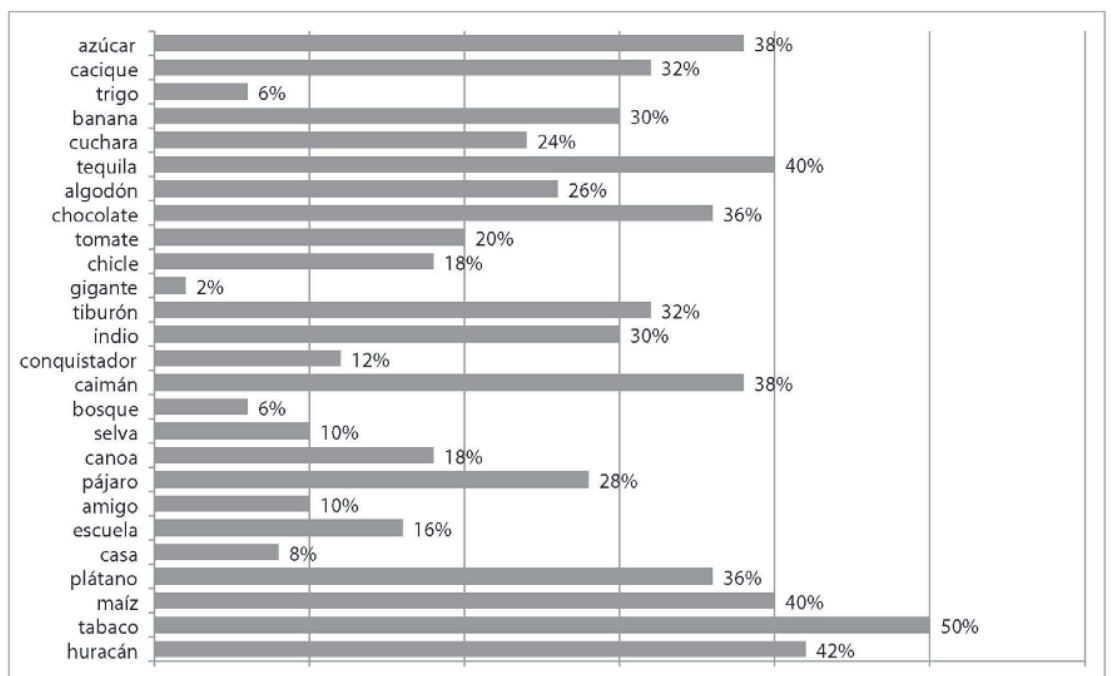

Gráfico 2: Respuestas a la pregunta «Elige las palabras que crees que son de origen hispanoamericano».

Con relación a los contactos del español con el náhuatl, se empezó por ver un vídeo relacionado con México, para adentrar al alumnado en el tema. Asimismo, el alumnado tuvo la oportunidad de ver el programa televisivo «Españoles por el Mundo: México D.F." y rellenó una tabla con información (lugares que hay que visitar, personas famosas, gastronomía, etc.). Además de los aspectos culturales, los alumnos señalaron las siguientes diferencias lingüísticas con relación: a) al léxico, como en pesero, chile, chipotle y mesero; b) al uso del prefijo rete-; c) al seseo y al yeísmo; y a d) algunas expresiones del habla coloquial, como «Ándele» $\mathrm{y}$ «Órale».

A continuación, a través de Pedro Páramo de Juan Rulfo, se trabajó la influencia del náhuatl en el español. Los alumnos leyeron algunos fragmentos de la obra y rellenaron una ficha de trabajo con ejercicios como los que vemos en la Figura 4. Estos ejercicios contienen algunas preguntas sobre las etapas de la historia, de los personajes, los símbolos, el tipo de lenguaje, las dimensiones de Comala en la novela, etc.

Los alumnos demostraron mucho interés por la obra, y fueron capaces de: buscar información sobre el autor y la historia de la novela; hacer un trabajo profundo con relación al léxico, buscando información en el diccionario de la RAE; aprender sobre la influencia del náhuatl, aunque demostrando dificultades, como podemos ver en las respuestas dadas por uno de los estudiantes en la Figura 4. Destacamos estos ejercicios, pues los alumnos buscaron el origen y el significado de las palabras que surgen en la novela, descubriendo, con la ayuda del diccionario español-náhuatl que encontramos en línea, que vienen del náhuatl. Además de las palabras que vemos en los ejercicios de la Figura 4, los alumnos tuvieron que descubrir los significados de vocablos como mitote, ocote, 
petaca, molote, chincual, atole y tilcuate. También tuvieron la oportunidad de reescribir expresiones como «nunca te platicó», "no le hace que nomás haya» y «Ese hijo de rechintola» en español peninsular.

D. Sustituye las palabra(s) subrayada(s) por otro término o expresión equivalente dentro del contexto. Busca los significados de dichas palabras o expresiones en DRAE

1. ...envenenado por el olor podrido de las saponarias jabonera (II planta herbácea)

2. Al llegar al infierno regresan por su cobija manta (II pieza para abrigarse).

3. hojas de toronjil, flores de Castilla, ramas de ruda

4. Nuestras madres nos parieron en un petate

5. Como usted sabe, no es fácil ajuarear las cosas en un dos por tres...

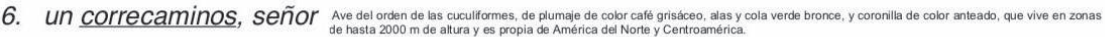

7. Pensaba en ti. Cuando volábamos papalotes en la época del aire... cometa de papel.

E. Busca el origen y el significado de las siguientes palabras. ¿A qué idioma pertenecen? usa este diccionario? http://www.gdn.unam.mx/termino/search. ¿Qué sabes de este idioma?

\begin{tabular}{|c|c|c|}
\hline Palabras & Origen & Definición/ Usos \\
\hline molcates & & Mazorca de maiz muy pequeña. \\
\hline \multirow[t]{2}{*}{ nixtenco } & & 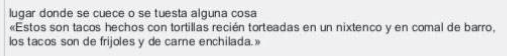 \\
\hline & & 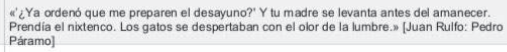 \\
\hline zopilote & Del nábuat tropilot. & 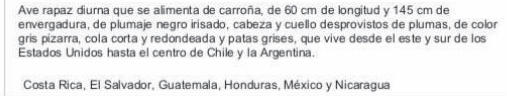 \\
\hline
\end{tabular}

Figura 4: Ejercicios hechos por uno de los estudiantes (No cambiamos las respuestas de los alumnos, por lo que pueden contener errores).

Como último ejercicio, los alumnos crearon un personaje, un murmullo, una voz de los muertos, para ser incluido en el cuento, teniendo en cuenta algunas palabras nahuas. En la Figura 5 vemos un ejemplo en el que la estudiante utilizó dos palabras: okichitli (hombre) e ikxitl (pie).

La propuesta didáctica siguiente está relacionada con los contactos de idiomas en el Cono Sur, más específicamente con el quechua y el guaraní. Como actividad de calentamiento y preparación del alumnado para la incursión en Hispanoamérica, se empezó la clase con un vídeo publicitario de la cadena comercial Sponsor ${ }^{3}$ y se activó el vocabulario necesario para el tema de la clase y la lectura del cuento Las medias de los flamencos de Quiroga.

$3<$ https://www.youtube.com/watch?v=IXUV0rNUhjA $>$. 
Yo caminaba, esperando ver algo más que las paredes oscuras y desnudas de las casas de la calle. E silencio daba miedo, y la obscuridad era fria. De repente, oi algo tenebroso, una corriente arrastrarse pelo suelo, como que aloo estaria atrapado y se intentase libertarse. Doble la esquina y lo vi. Un okichti alto y delgado, pálido y viejo, sus ojos negros y fríos. Su pelo era rubio y desordenado. Sus ropas eran de lujo, pero estaban rasgadas y sucias, y él caminaba con la ayuda de una bengala. Una corriente estaba presa en vuelta de su ikooti derecho, y arrastraba atrás de si un cáliz de oro y piedras preciosas, una bolsa con tomin, y una calavera de oro con cuernos y colmillos. Cuando me vio, me preguntó:

"¿Qué pasa, chico? No me mires asi. ¿No me conoces?" "Perdóname, sefior.., pero no sé quién usted es."

"¿Cómo? Yo soy Esteban Julio Montoya de la Rosa Ramirez, hijo de Adelardo Benedito Julio Montoya de la Rosa Ramírez, señor de estas tierras. iUsted tiene de mostrar respeto!" y levantó su nariz.

"Señor Ramirez."

"iNo! Yo soy Esteban Julio Montoya de la Rosa Ramirez, hijo de Adelardo Benedito Julio Montoya de la Rosa Ramirez, señor de estas tierras. iMuestre respetol " y se fue.

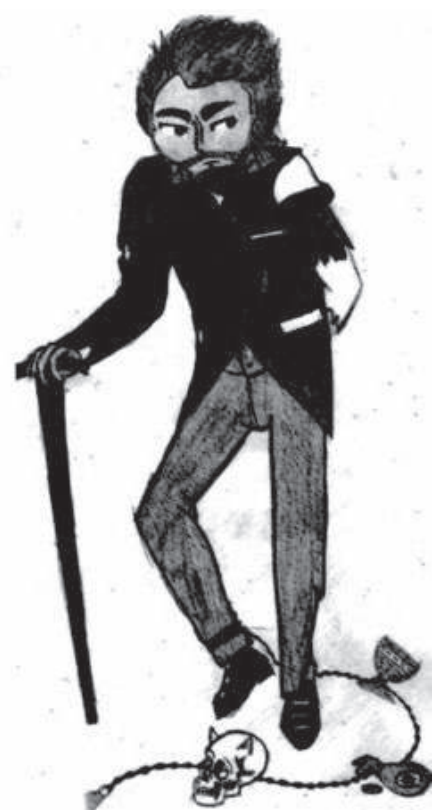

Figura 5: Murmullo creado por uno de los estudiantes.

Los alumnos tuvieron la oportunidad de revisar las prendas de vestir de una manera divertida y tomar contacto con la variedad andina, con relación al acento y a expresiones coloquiales (;Qué bacán!, hacer lo bien sin mirar a quien, etc.) Se revisó el léxico relacionado con las prendas de vestir con la aplicación Heads $U p !$. Seguidamente, los alumnos también crearon un mapa mental con el léxico que ya conocían en el sitio web GoConqr ${ }^{5}$ (véase la Figura 6).

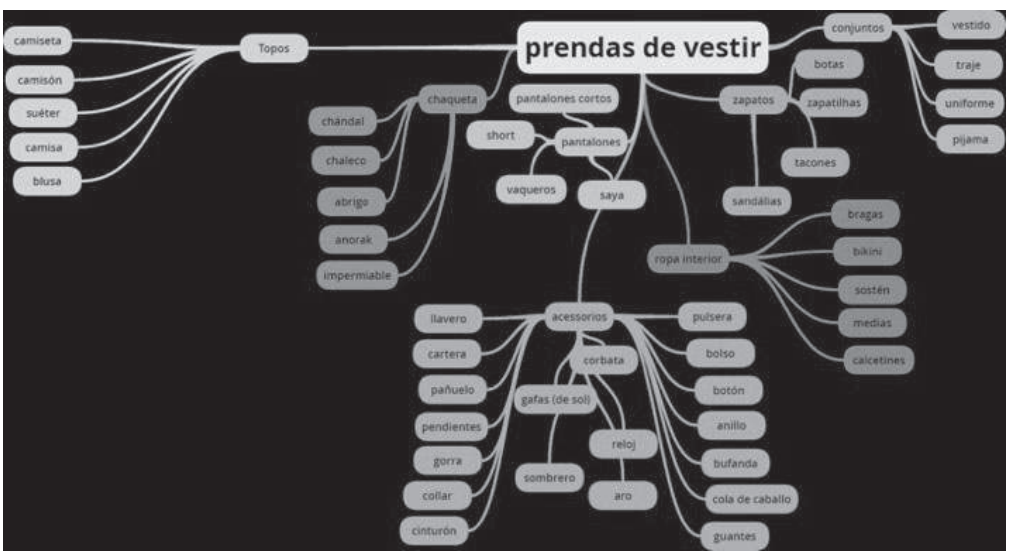

Figura 6: Ejemplo de un mapa mental creado por uno de los alumnos.

4 <https://play.google.com/store/apps/details?id=com.wb.headsup\&hl=pt> $<$ https://itunes.apple.com/us/app/heads-up/id623592465? $\mathrm{mt}=8>$.

$5<$ www.goconqr.com $>$. 
Después de leer el cuento en plenaria, se hicieron algunas preguntas sencillas sobre su contenido, como las siguientes: ejercicios de verdadero o falso, preguntas de comprensión un poco más complejas, ejercicios de búsqueda de significado de las palabras y su origen en el Diccionario de la Lengua Española, entre otros (véase la Figura 7). De este modo, los alumnos tomaron contacto con la variedad rioplatense y descubrieron que el español es un idioma que contiene términos que surgieron del guaraní.

\section{Durante la lectura}

A. Lee el texto.

B. Intenta responder a las siguientes preguntas. Contesta "Verdadero" o "Falso" y corrige las frases falsas.

1. Según el texto, los peces estaban un poco bebidos y no bailaron.

2. Los yacarés se habían adornado bien con collares.

3. Los sapos y las ranas caminaban durante la fiesta.

4. Las víboras iban muy bonitas y llevaban trajes de bailarina combinada con su color.

5. A los flamencos les resultó difícil convencer a la lechuza.

6. El cuervo les dice que bailen todo el rato.

7. Durante el baile las víboras empezaron a sospechar del estratagema.

8. Por las mordidas, murieron muchos flamencos.

9. Según el texto, los flamencos no tienen un pelo de tontos.

10. Los flamencos siguen sintiendo dolor aún hoy y intentan curarse en el agua.

D. Contesta a las preguntas siguientes de manera completa.

1. ¿Cuál era el objetivo de los flamencos?

2. ¿Cuál es la recomendación que les hace la lechuza a los flamencos cuando les entrega las medias? ¿Para qué les hace esa recomendación?

3. ¿Qué acción da inicio al conflicto del cuento?

4. ¿En qué lugar se desarrollan los hechos? Señala en el texto los fragmentos que lo justifiquen.

5. ¿Por qué se enfurecieron las víboras?

6. ¿Cuál fue el error de los flamencos? ¿Son culpables los flamencos? ¿Por qué?

7. ¿Qué intenta explicar el cuento? ¿Qué enseñanza puedes extraer del cuento?

C. Busca las siguientes palabras en el diccionario de la RAE y escribe su significado. Algunas de las palabras provienen de otro idioma. ¿Qué lengua es?

\begin{tabular}{|l|l|}
\hline Palabra & \multicolumn{1}{|c|}{ Significado } \\
\hline 1. Yacarès & \\
\hline 2. Orilla & \\
\hline 3. Asomados & \\
\hline 4. Bananas & \\
\hline
\end{tabular}

Figura 7: Ejercicios de comprensión del cuento «Las medias de los flamencos».

Después de la lectura, los alumnos tuvieron la oportunidad de (re)crear el cuento usando el sitio PowToon ${ }^{6}$, en el cual elaboraron un vídeo imaginando un

$6<$ https://www.powtoon.com/home/?>. 
encuentro entre los flamencos y la lechuza después de la fiesta ${ }^{7}$. A través de este ejercicio se estimula la creatividad de los alumnos, pues tuvieron la oportunidad de relacionarse con el texto de otra manera (Fonseca 1992; Mercau 2014).

Además, como tarea final, los alumnos también podían ampliar sus mapas mentales, los cuales habían creado en el GoConqr, añadiendo nuevas palabras que encontraron en los catálogos de ropa de las regiones andina o rioplatense, consultando a los sitios Sabrina y Patprimo 8 e incluyendo palabras como remera, campera y guyambera.

Como última propuesta didáctica, nos fijaremos en la influencia del italiano en el español argentino a través de un fragmento ${ }^{9}$ de la película Relatos Salvajes. Se empezó la clase con la consulta del blog Diario del viajero ${ }^{10}$. Con esta actividad los alumnos (re)activaron el vocabulario necesario para la comprensión del fragmento y (re)descubrieron la historia subyacente detrás de palabras como boleto, billete, chárteres, chequear, maleta, petaca, visa, visado, rellenando una tabla con información acerca de su origen y usos. Después del visionado, se pidió a los estudiantes que se fijasen en la conversación entre los viajeros protagonistas ${ }^{11}$ en el fragmento (véase la Figura 8).

Seguidamente, los alumnos se concentraron en los pronombres personales y los verbos, subrayándolos y reflexionando sobre su rol en el diálogo establecido. La mayoría de los alumnos identificó el voseo, y luego se les pidió que analizaran los artículos Por qué algunos países de América Latina usan el 'vos' en vez del 'tú? ${ }^{12}$ y Até que o vós me doa ${ }^{13}$, que explican el uso y el origen del vos y del você en portugués, permitiéndoles hacer un análisis contrastivo y comprender su origen, rasgos y usos geográficos.

También se aprovechó el fragmento para trabajar las características fonéticas de la variedad del español rioplatense, por lo que los estudiantes vieron de nuevo el fragmento y tomaron nota de ejemplos que escucharon para cada uno de los rasgos fonéticos (véase la Figura 9), incluyendo el yeísmo, la aspiración

7 Consultable en <https://www.powtoon.com/online-presentation/bvgG7kL4pt9/?utm _campaign=email\%2Bshare\%2Bby\%2Bowner\&utm_content=bvgG7kL4pt9\&utm _ po $=13750663 \& u t m \_s o u r c e=$ player-page-social-share\&utm_medium=SocialShare \&mode $=$ movie $>$.

8 Se puede acceder a estos catálogos de ropa a través de los enlaces siguientes: $<w w w$. sabrina.com.ar y http://www.patprimo.com>.

$9<$ https://www.youtube.com/watch?v=nYrMRcnqBn8>.

$10<\mathrm{https} / /$ www.diariodelviajero.com/tag/etimologias-viajeras $>$.

11 «Usted, ¿qué hace?/ Me mataste./ ¿Por qué?/ Ese usted me hundió hasta el quinto círculo del infierno. Tengo que hacerme un entretejido urgente. [...]/ ¿Ahora la querés arreglar? [...]/ Vos elegí el término que quiera y yo lo instalo en la comunidad artística. [...]/ ¿Lo seguís viendo?»

$12<$ http://www.bbc.com/mundo/noticias-america-latina-36928497>.

$13<$ http://visao.sapo.pt/opiniao/ricardo-araujo-pereira/2016-12-15-Ate-que-o-vos-medoa $>$. 
frecuente de la /s/, la emisión frecuente de la $s$ o de la $r$ al final de una palabra y la pronunciación con semejanzas del italiano.

B. ¿Verdadero or Falso? Elige la opción correcta y corrige las frases incorrectas.

\begin{tabular}{|l|l|}
\hline & V F \\
\hline 1. La viajera consigue las millas a su nombre. & \\
\hline 2. La azafata le dice que están embarcando por la puerta trece. \\
\hline 3. La viajera no necesita de ayuda con el equipaje de cabina. \\
\hline 4. La viajera es una modelo de publicidad. \\
\hline 5. El viajero se dedica a la crítica musical. \\
\hline 6. Los dos están en un asiento de ventanilla. \\
\hline 7. Ellos se dan cuenta de que conocen a Gabriel Pasternak. \\
\hline 8. La madre de Gabriel Pasternak también está en la aeronave. \\
\hline 9. Uno de los pasajeros intentó cambiar la fecha de viaje. \\
\hline 10. La azafata está muy tranquila.
\end{tabular}

C. Contesta a las preguntas.

1. ¿Crees que el crítico musical está coqueteando con la modelo? Justifica.

2. ¿Cuál es la relación de los pasajeros con Gabriel Pasternak?

3. ¿Cómo les burló Gabriel Pasternak?

Figura 8: Ejercicios relacionados con el fragmento de Relatos Salvajes.

F. En la película escuchamos la variedad del español argentino. Te suena un poco diferente del español peninsular, ¿verdad? Ve de nuevo el fragmento y transcribe algunos ejemplos.

\begin{tabular}{|l|l|}
\hline Rasgos fonéticos & Ejemplos \\
\hline $\begin{array}{l}\text { el yeísmo con rehilamiento o sea la /ly la } y \text { se } \\
\text { pronuncian como /sh/ }\end{array}$ & \\
\hline $\begin{array}{l}\text { la aspiración frecuente de la /s/, que sucede cuando } \\
\text { la /s/ se pronuncia como [h] }\end{array}$ \\
\hline la elisión frecuente del [s] o [r] final de una palabra \\
\hline $\begin{array}{l}\text { la pronunciación presenta semejanzas con el } \\
\text { italiano }\end{array}$ \\
\hline el seseo (pronuncian $<\mathrm{s}>,<\mathrm{c}>\mathrm{y}<\mathrm{z}$ como [s]
\end{tabular}

Figura 9: Ejercicio relacionado con los rasgos fonéticos del español argentino. 


\section{Conclusión: resultados y consideraciones finales}

Las secuencias didácticas que creamos contienen muestras lingüísticas y culturales vivas (Lerner 2000) de las variedades del español de una manera integrada y, con ellas, se busca involucrar al alumnado en su análisis lingüístico y cultural.

Creemos que estas propuestas didácticas ayudan al alumnado a reflexionar sobre el tema de los contactos de lenguas en la clase de ELE, valorando la diversidad lingüística como un concepto clave para la comprensión de la cultura que subyace detrás de los idiomas que hablamos hoy.

Creemos que el aprendizaje de ELE debe ser una oportunidad para desarrollar una relación de afectividad con el lenguaje y sus culturas (Marques 2004; Instituto Cervantes 2006), permitiendo la creación de situaciones de interacción, recepción e interpretación de diversos tipos de discursos, con respecto a una variedad de tipos de textos literarios e hipermedia (Cruz 2017; Cruz y Saracho 2018), como los que vimos en este artículo. Con un enfoque comunicativo experiencial y una pedagogía de los discursos, los alumnos interactúan con los textos y los recrean, descubriendo los rasgos lingüísticos y culturales de las distintas variedades del español.

\section{Bibliografía}

Altmann W. y Vences U., 2004, América latina en la enseñanza del español. Encuentro o encontronazo? Theorie und Praxis des moderen Spanischunterrichts, Berlín, Edition Tranvia.

Anadón Pérez M., 2003, Hispanoamérica y el español de América en la enseñanza del español como segunda lengua entre alumnos ingleses, Barcelona, Universidad de Barcelona.

Azorín Fernández D., 2008, «Los americanismos en El Nuevo Diccionario de la lengua castellana», Revista de Investigación Lingüística, 11, p. 13-43.

Boyd-Bowman P., 1976, «Patterns of Spanish emigration until 1600», Hispanic American Historical Review, 56, p. 580-604.

Canfield D., 1934, Spanish literature in Mexican languages as a source for the study of Spanish pronunciation, Nueva York, Instituto de las Españas en los Estados Unidos.

Castillo I., 1965, México y su revolución educativa, México DF, Academia Mexicana de la Educación.

Cerrón Palomino R., 1987, Lingüírstica quechua, Cuzco, Centro de Estudios Rurales Andinos «Bartolomé de las Casas».

Colantoni L., 2004, La huella del italiano persiste en el español de la Argentina. Unidad en la diversidad, Portal informativo de la lengua castellana. 
Company Company C., 2016, Historia del español en América. in GutiérrezRexach J. (ed.), Enciclopedia de lingüística Hispánica, Nueva York, Routledge, p. 601-612.

Cruz M., 2017, «Variación diatópica del español americano a través de recursos hipermedia». Revista Comunicación, 26, p. 1-26.

Cruz M. y Saracho M., 2018, «La variación diatópica del español de América a través de una (hiper)pedagogía plurilingüística e intercultural», Revista Diacrítica-Número especial dedicado al VII Congreso sobre la enseñanza del Español Lengua Extranjera en Portugal, p. 133-153.

Fernández-Corbacho A., 2014, "Aprender una Segunda Líengua desde un Enfoque Comunicativo Experiencial», Programa de Desarrollo Profesional: El enfoque comunicativo experiencial: claves para un aprendizaje duradero.

Flores Farfán J., 1998, "On the Spanish of the nahuas», Hispanic Linguistics, 10, p. 1-41.

Foncubierta J. y Rodríguez C., 2015, Didáctica de la gamificación en la clase de español. Programa de Desarrollo Profesional, Madrid, Editorial Edinumen.

Fonseca F., 2000, «Da inseparabilidade entre o ensino da língua e o ensino da literatura», in Reis C. (ed.), Didáctica da língua e da literatura, Coimbra, Almedina, p. 37-45.

Fonseca F.I., 1986, "Competência narrativa e ensino da língua materna», Palavras, 9, p. 6-10.

Fonseca J., 1992, "Ensino da língua materna como pedagogia dos discursos», in Linguística e Texto/Discurso. Teoria, Descrição, Aplicação. Lisboa/Niza, Ministério da Educação/ Universidad de Niza, p. 235-248.

Fontanella de Weinberg M., 1992, El español de América, Madrid, Mapfre.

Gómez R., 2003, Sociolinguistic correlations in the Spanish spoken in the Andean region of Ecuador in the speech of the younger generation, Toronto, University of Toronto.

Granda G., 1980, «Préstamos morfológicos del guaraní en el español del Paraguay», Revue de linguistique romane, 44, p. 57-68.

Heath S., 1972, La política del lenguaje en México: de la Colonia a la Nación, México DF, INI.

Instituto Cervantes I., 2006, Plan Curricular del Instituto Cervantes. Niveles de referencia para el español, Madrid, Instituto Cervantes-Biblioteca Nueva.

Klee C. y Lynch A., 2009, El español en contacto con otras lenguas, Washington, Georgetown University Press.

Knutson S., 2015, «Experiential Learning in Second Language Classrooms». TESL CANADA 20.

León-Portilla M., 2002, El español y las lenguas amerindias. Un patrimonio intangible, <https://www.letraslibres.com/mexico/el-espanol-y-las-lenguasamerindias-un-patrimonio-intangible>, consultado el 03/06/2019.

Lipski J., 1994, El español de América, Madrid, Cátedra. 
Lockart J., 1991, Nahuas and Spaniards: postconquest central Mexican history and philology, Stanford, Stanford University Press.

Lope Blanch J., 1967, «La influencia del sustrato en la fonética del español de México», Revista de Filología Española, 50, p. 145-161.

Marques L., 2004, «O aproveitamento didáctico da canção na aula de língua estrangeira», A Página da Educação, 130.

Mercau C., 2014, La cultura hispanoamericana en las clases de ELE: nuevas perspectivas y aplicaciones, Oviedo, Universidad de Oviedo.

Mora C., 2013, Gamificación: lo congitivo, lo emocional y lo social, <https:// gamificationedufis.wordpress.com/2015/06/09/lo-cognitivo-lo-emocional-ylo-social/>.

MorenoFernándezF., 2010, Lalenguaespañolaensugeografía, Madrid, Arco Libros, P21, 2015, P21 Framework Definitions, <https://eric.ed.gov/?id=ED519462>.

Parodi C., 2006, «The Indianization of Spaniards in New Spain», in Hidalgo M. (ed.), Mexican indigenous languages at the dawn of the twenty-first century, Berlínn, Mouton de Gruyter, p. 29-52.

Sánchez-Albornoz N., 2006, Rumbo a América. Gente, ideas y lengua, México, Colegio de México.

Shams L. y Seitz A., 2008, «Benefits of multisensory learning», Trends in Cognitive Sciences, 12, p. 411-417.

Teruggi M., 1978, Génesis y esencia de las hablas coloquiales urbanas, Buenos Aires, Editorial Sudamericana. 